\begin{tabular}{|c|l|}
\hline Title & Chlamydia trachomatis serovar L2 infection model using human Iymphoid Jurkat cells \\
\hline Author(s) & $\begin{array}{l}\text { Kubo, Takeru; Ishida, Kasumi; Matsuo, Junji; Nakamura, Shinji; Hay ashi, Y asuhiro; Sakai, Haruna; Y oshida, } \\
\text { Mitsutaka; Takahashi, Kaori; Hirai, Itaru; Y amamoto, Y oshimasa; Y amaguchi, Hiroyuki }\end{array}$ \\
\hline Citation & $\begin{array}{l}\text { Microbial Pathogenesis, 53(1), 1-11 } \\
\text { https://doi.org/10.1016j.micpath.2012.02.005 }\end{array}$ \\
\hline Issue Date & 2012-07 \\
\hline Doc URL & http://hdl.handle.net/2115/49575 \\
\hline Type & article (author version) \\
\hline File Information & MP531_1-11.pdf \\
\hline
\end{tabular}

Instructions for use 


\section{Chlamydia trachomatis serovar L2 infection model using human lymphoid}

\section{Jurkat cells}

Takeru Kubo $^{\mathrm{a}}$, Kasumi Ishida ${ }^{\mathrm{a}}$, Junji Matsuo ${ }^{\mathrm{a}}$, Shinji Nakamura ${ }^{\mathrm{b}}$, Yasuhiro Hayashi ${ }^{\mathrm{a}}$, Haruna Sakai ${ }^{\mathrm{b}}$, Mitsutaka Yoshida $^{\mathrm{b}}$, Kaori Takahashi ${ }^{\mathrm{b}}$, Itaru Hirai $^{\mathrm{c}}$, Yoshimasa Yamamoto $^{\mathrm{c}}$ and Hiroyuki Yamaguchi ${ }^{\mathrm{a}}$ *

${ }^{a}$ Department of Medical Laboratory Sciences, Faculty of Health Sciences, Hokkaido University, Nishi-5 Kita-12 Jo, Kita-ku, Sapporo, Hokkaido 060-0812, Japan

${ }^{b}$ Division of Biomedical Imaging Research, Juntendo University Graduate School of Medicine, 2-1-1 Hongo, Bunkyo-ku, Tokyo 113-8421, Japan

${ }^{c}$ Laboratory of Molecular Microbiology, Department of Bioinformatics, Graduate

School of Medicine, Osaka University, 1-7 Yamadaoka, Suita, Osaka 565-0871, Japan

*Corresponding author.

Mailing address: Department of Medical Laboratory Science, Faculty of Health Sciences, Hokkaido University, Nishi-5 Kita-12 Jo, Kita-ku, Sapporo, Hokkaido 060-0812, Japan

Phone: +81-11-706-3326; Fax: +81-11-706-3326

E-mail: hiroyuki@med.hokudai.ac.jp 


\section{Abstract}

Chlamydia trachomatis L2 invasively attacks lymphatic and subepithelial tissues of the genital tract during the formation of primary lesions. This subsequently results in lymphadenopathy, and suggests a greater propensity for systemic dissemination. However, whether lymphocytes are a potential vehicle cell for the dissemination of this infection remains unknown. We therefore assessed the growth properties of $C$. trachomatis L2 in lymphoid Jurkat cells compared with those observed in epithelial HeLa cells. Both cells supported the growth of $C$. trachomatis with a similar increase in infective progenies. Enriched human-blood lymphocytes also supported the $C$. trachomatis growth as well as Jurkat cells. Bacteria infecting the Jurkat cells were more susceptible to antibiotics (doxycycline, azithromycin, ofloxacin) than those in HeLa cells. Of the sphingomyelin biosynthesis inhibitors tested, both myriocin and fumonisin B1 significantly inhibited bacterial growth in both cells types. A Jurkat cell mutant that impaired bacterial growth was established using ethylmethanesulfonate treatment. DNA microarray analysis with real-time reverse transcription-polymerase chain reaction revealed that the mutant cells over-expressed granzyme $\mathrm{K}$ gene. Immunofluorescence staining also indicated that granzyme $\mathrm{K}$ irregularly over-expressed among the mutant cells as compared with that of the wild cells, suggesting a possible mechanism refractory to $C$. trachomatis infection. Thus, we concluded that $C$. trachomatis $\mathrm{L} 2$ could infect Jurkat cells with lymphoid properties, providing a new tool for studying $C$. trachomatis dissemination to tissues via lymphocyte movement.

Keywords: Chlamydia trachomatis; serovar L2; Lymphocytes; Jurkat cells; Sphingolipid; Ethylmethanesulfonate 


\section{Introduction}

The obligate intracellular bacterium, Chlamydia trachomatis, is the leading cause of ocular and genital infections worldwide. Usually asymptomatic, untreated disease can sometimes progress to chronic infections that include serious sequelae. Chronic ocular infections due to serovars $\mathrm{A}$ to $\mathrm{C}$ result in trachoma, and are the second highest cause of preventable blindness [1, 2]. More prevalent are the chronic genital infections with serovars $\mathrm{D}$ to $\mathrm{K}$, which are responsible for pelvic inflammatory diseases, ectopic pregnancy and tubal infertility in women [3, 4]. While C. trachomatis serovars L1-3, cause systemic diseases such as lymphogranuloma venereum (LGV) [5]. LGV is considered a sporadic disease occurring worldwide particularly in men who have sex with men [5-12]. LGV is an invasive disease caused predominantly by $C$. trachomatis serovar L2, and unlike other genital C. trachomatis infection, preferentially affects lymphatic tissue after invasion through an epithelial surface [5-12]. Thus, $C$. trachomatis L2 may potentially transfer from the epithelial surface to lymphatic tissue via unknown vehicle cells, possibly associating with systemic diseases produced by this bacterial serovar.

Chlamydiae have a unique developmental cycle that is not present in any other bacterial families, and is characterized by two functionally and morphologically distinct bacterial forms, the reticulate body (RB) and elementary body (EB) [13-15]. Infection is initiated by the metabolically inactive EB that subsequently differentiates into the larger, metabolically active RB after endocytosis [13-15]. Maturation further occurs in the surrounding plasma membrane, known as an inclusion [13-15]. Recently, our studies 
Kubo Tet al

demonstrated that Chlamydophila pneumoniae, a species closely related to $C$. trachomatis, could infect and multiply in the human lymphocyte cell line Molt-4 and mouse spleen lymphocytes, thereby completing its developmental cycle $[16,17]$. Furthermore, Kaul et al. identified $C$. pneumoniae deoxyribonucleic acid (DNA) in $\mathrm{CD}^{+}$lymphocytes obtained from cardiology patients [18]. Thus, lymphocytes are a potential host cell for not only $C$. pneumoniae, but also $C$. trachomatis infection.

In this study, we aimed to compare the growth traits of $C$. trachomatis L2 in lymphoid Jurkat cells with those in epithelial HeLa cells.

\section{Results}

2. 1. Growth of C. trachomatis L2 in lymphoid Jurkat cells and enriched human-blood lymphocytes, followed by successful infection

C. trachomatis was confirmed to grow in both Jurkat and HeLa cells using one-step growth curve analysis, fluorescence staining and electron microscopy. Cells were infected with bacteria and incubated for up to seven days. Bacteria appeared to grow normally within typical inclusions for both cell types [Fig. 1A (HeLa) and B (Jurkat)]. Fluorescence staining data showed that an increase in inclusion size that was dependent on the culture period occurred in the Jurkat cells, which also supported the evidence of normal C. trachomatis growth in this cell type (Fig. 1C). Also, transmission electron microscopic (TEM) analysis revealed normal growth in inclusion formed into Jurkat cell, even with a few aberrant bodies $(\mathrm{AB})$, which is a representative morphological 
form in persistent infection (Jurkat, Fig. 2; HeLa, [19-20]). In addition, we also confirmed that enriched human-blood lymphocytes obtained from three healthy donors could support the C. trachomatis growth (Fig. 3).

\section{2. Susceptibility of $\mathrm{C}$. trachomatis L2 to antibiotics in lymphoid Jurkat cells}

To confirm whether the bacteria developed into a stage of persistent infection, we assessed the susceptibility of $C$. trachomatis to antibiotics [doxycycline (DOX), azithromycin (AZM), ofloxacin (OFLX)] during Jurkat cell infection. Assessment with IFU assay showed that all antibiotics used for this study were very effective against $C$. trachomatis L2 growth in Jurkat cells as well as HeLa cells (Fig. 4A). It is further intriguing that the bacteria appeared to be more sensitive in the Jurkat cells than in HeLa cells, although C. pneumoniae was previously reported to strongly resist antibiotics in human lymphoid Molt-4 and P3HR1 cells [22]. Thus, the results obtained indicated that $C$. trachomatis L2 in lymphoid Jurkat cells was susceptible to antibiotics, to a similar or greater level than that observed in epithelial HeLa cells, suggesting that bacteria normally could proliferate within these cells. Both the results of immunofluorescence staining of inclusion and real-time reverse transcription (RT)-polymerase chain reaction (PCR) targeting to the bacterial $16 S r R N A$ also ensured that the bacteria were very sensitive to the antibiotics used for study in Jurkat cells, supporting the bacterial complete maturation in the cells (Fig. 4B and C).

\section{3. Sphingomyelin acquisition of $\mathrm{C}$. trachomatis L2 in lymphoid Jurkat cells}

Because it is well known that host cell sphingolipids are required for the intracellular growth of $C$. trachomatis [23-25], sphingomyelin acquisition by $C$. trachomatis L2 in 
Jurkat and HeLa cells was compared by using two different specific inhibitors of sphingomyelin biosynthesis in cells, myriocin and fumonisin B1. Myriocin is the initial enzyme in the biosynthesis of sphingomyelin via the endoplasmic reticulum (ER), and fumonisin B1 is a drug that can not only inhibit ceramide synthesis from sphingosine in the Golgi, but also block dihydroceramide synthesis from sphinganine at ER [23-25]. We found that both drug significantly inhibited the infectious progenies of $C$. trachomatis L2 in both cells (Fig. 5).

\section{3. Establishment of mutant Jurkat cells resistant to C. trachomatis infection and its} characterization

We successfully established and isolated a mutant clone derived from Jurkat cells that had become resistant to chlamydial infection, by using conducting random chemical mutagenesis with EMS as the mutagen to assess infection differences between Jurkat and HeLa cells. Representative images showed that infection had successfully occurred within the wild cells, while it appeared that the bacteria failed to grow within the mutant cells (Fig. 6A). No significant difference in C. trachomatis L2 infectious rate was observed between to mutant cells and wild cells (Fig. 6B), demonstrating that the molecules associated with bacterial attachment to the cells did not change as a consequence of mutagenesis. In contrast, bacterial growth in the mutant cells significantly decreased compared with that in the wild cells (Fig. 6C), suggesting potential impairment of certain host cell molecules directly associated with bacterial development. We performed a similar experiment with $C$. trachomatis $\mathrm{D}$, the growth of the bacteria in the mutant cells significantly decreased, which was also the case for $C$. trachomatis L2 (Fig. 6D). We also confirmed gene expression changes in the mutant 
cells as compared with the wild cells by using DNA microarray analysis. As a result, the DNA microarray analysis revealed a considerable increase in the granzyme K gene (14 fold vs. the parent cells), and a decrease in the histone cluster $1 \mathrm{H} 4 \mathrm{c}$ gene $(0.03$ fold vs. the parent cells) in the mutant cells (Fig. 7). We also confirmed obvious changes of these gene expressions by real-time RT-PCR with a strong expression of granzyme K in the mutant cells as expected (Fig. 8). Moreover, immunofluorescence staining of granzyme $\mathrm{K}$ interestingly revealed irregular over-expression of the protein among the mutant cells of approximately $13 \%$ as compared with the wild cells; eventually any irregular expression changes of granzyme $\mathrm{K}$ in the wild cells was not seen (Fig. 9).

\section{Discussion}

We previously reported that of the pathogenic chlamydiae $C$. pneumoniae could infect lymphocytes, producing a down-regulation in CD3 expression, which is a critical ligand for antigen-presenting cells, or CD25 expression, which is also a critical receptor for detecting immune stimulation [26, 27]. These findings suggest that immune functions of lymphocytes infected with $C$. pneumoniae may be altered, resulting in an inappropriate immune response to stimulation that eventually contributes to chlamydiae pathogenesis. Hence, investigating the attachment mechanism of $C$. pneumoniae to human lymphocytes is critical for better understanding the pathogen's role in complex disease

pathogenesis. We further demonstrated that the $C$. pneumoniae mechanism of lymphocyte attachment is independent of heparin, which is generally utilized for bacterial attachment to epithelial cells in a Jurkat cell model [28]. These cells are a 
well-characterized human lymphocyte cell line, and have representative traits of primary human lymphocytes [29]. Thus, at least one of the pathogenic chlamydiae, $C$. pneumoniae, has a tropism to lymphocytes achieving successful infection via intriguing mechanisms. This also suggests lymphocytes are a potential host cell for not only $C$. pneumoniae but also $C$. trachomatis infection, which particularly for serovar L2 could disperse from local mucosal tissue to lymphoid tissue. Furthermore, a recent work indicated that $C$. trachomatis induces anti-inflammatory effect in human macrophages by attenuation of immune mediators in Jurkat T-cells cultured with the bacteria [30], implying successful infection of $C$. trachomatis L2 to Jurkat cells. However, whether lymphocytes are a potential vehicle cell for $C$. trachomatis L2 dissemination still remains unknown. We therefore assessed the growth properties of $C$. trachomatis $\mathrm{L} 2$ in lymphoid Jurkat cells in comparison with that observed in epithelial HeLa cells and showed that $C$. trachomatis L2 could infect cells with lymphoid properties.

One-step growth curves based on fluorescence microscope analysis supported that the normal bacterial growth observed in Jurkat cells was similar to that in HeLa cells, although bacterial growth in Jurkat cells was likely to be slightly delayed. It is well known that sequestered inclusions of $C$. trachomatis need to acquire nutrients by fusing with multivesicular bodies (MVBs) or vesicles present in the cytoplasm of infected cells $[31,32]$. Although the delayed mechanism remains unknown, it could not be denied that accessibility to MVBs or vesicles differs among host cell types. We recently demonstrated that heparin expression on Jurkat cells, available for the attachment of pathogenic chlamydiae to epithelial host cells, was undetectable [28], indicating that $C$. trachomatis L2 attachment to Jurkat cells is potentially heparin-independent. Moreover, 
although it has been believed that prolonged incubation of EBs in culture media leads to loss infectivity, it was surprisingly to find that no obvious decrease of the number of the infectious progenies in the cultures was seen at a time point of 7-day post infection. The results imply that more than our expectation, EB may be a stable form, supported by our previous data that relative pathogenic chlamydia $C$. pneumoniae could survive at least a month in cell-free culture condition [33].

TEM analysis of $C$. trachomatis L2 infected Jurkat cells clearly revealed that the bacteria were located in large cytoplasmic inclusions within the infected cells $48 \mathrm{~h}$ after infection, demonstrating successful bacterial replication and growth. Individual bacterial growth stages differed among each of the inclusions including EBs and RBs with a few ABs, which was not similar to more synchronized growth previously reported during late stage bacterial growth in infected HeLa cells [34, 35]. Such chlamydial aberrant bodies could be induced by exposure to antimicrobial agents or cytokines such as IFN $\gamma$ [36-38]. However, no IFN $\gamma$-expression and production was found on the infected Jurkat cells in our study (data not shown). Although this unsynchronized mechanism in Jurkat cells should be clarified, it is possible this provides an advantage for pathogenic chlamydial survival against various host pressures used to eliminate the bacteria.

It is notable that enriched human-blood lymphocytes allow $C$. trachomatis L2 infection, suggesting a possibility that primary lymphocytes may be a suitable host cells for supporting the bacterial growth in vivo. Although the donor numbers were limited, the results obtained from the experiment with primary cells certainly supported our data 
regarding $C$. trachomatis $\mathrm{L} 2$ infection to human lymphoid cell line, Jurkat cells.

Jurkat cells were clearly capable of supporting all C. trachomatis L2 life stages in an inclusion, with maturation from RB to EB following secondary infection. This implies that the bacteria in metabolically active stages are more susceptible to antibiotics than the bacteria entering the dormant phase of persistent infection. To confirm this possibility, we found that all antibiotics used for this study were very effective against $C$. trachomatis L2 growth in Jurkat cells, suggesting successful bacterial growth occurred in Jurkat and epithelial cells. We previously reported that C. pneumoniae is strongly resistant against antibiotics in human lymphoid Molt-4 and P3HR1 cells, in addition to mouse primary splenocytes [22]. This is in contrast with the results observed for $C$. trachomatis L2 in Jurkat cells, implying C. pneumoniae infecting lymphocytes easily enters a persistent, yet viable, stage that lacks metabolic activities, and consequently antibiotics could not eliminate the bacteria from the host cells.

Also, it is intriguing that $C$. trachomatis L2 in Jurkat cells appear to be more sensitive to antibiotics than in HeLa cells. Although the mechanism for this remains unknown, it is possible that the antibiotics entering the host cell cytoplasm might be very effective because of the narrow cytoplasmic space of the lymphocyte when compared with that for epithelial cells. Moreover, the susceptibility to antibiotics of C. trachomatis L2 growing in lymphocytes indicate that antibiotic treatment would prevent the progenies of infection to advanced sequelae such as pelvic inflammatory disease.

While it is believed that host-parasite interactions allow for the acquisition of 
biosynthetic precursors such as amino acids and lipids from host cells to parasites, it is clear that eukaryotic host cell-derived sphingomyelin is essential for intracellular growth of $C$. trachomatis [23, 31, 32, 39]. Therefore, we examined whether the sphingomyelin biosynthetic pathway required for $C$. trachomatis $\mathrm{L} 2$ growth differs between Jurkat and HeLa cells. The sphingomyelin biosynthetic pathway consists of two different steps, whereby the precursors of sphingomyelin are synthesized in the endoplasmic reticulum (ER) and the final step involves the subsequent transfer of ceramide to the Golgi apparatus [39]. Therefore, we used two different inhibitors, myriocin, which inhibits the synthesis of the sphingomyelin precursor in the ER, and fumonisin B1, which not only inhibits the final step sphingomyelin synthesis in the Golgi apparatus, but also block dihydroceramide synthesis from sphinganine at ER [39]. As a result, both drugs significantly inhibited the infectious progenies of $C$. trachomatis L2 in both cells in the same way, suggesting that sphingomyelin acquisition of $C$. trachomatis L2 in lymphoid Jurkat cells might be similar to that of the bacteria in epithelial HeLa cells. Although so far it has been shown a novel way for sphingomyelin acquisition based on the interaction of lipid transfer protein CERT (ceramide transfer protein) with chlamydial IncD protein at ER-chlamydial inclusion membrane contact site [40], the association of the bacterial growth in lymphocytes with this way remains undetermined.

Furthermore, we successfully established and isolated a mutant clone derived from Jurkat cells that was resistant to chlamydial infection in an attempt to determine host factors specific to lymphocytes that are involved in supporting of chlamydial growth. While the mutant cells did not reduce $C$. trachomatis L2 attachment as much as for 
other serovar D bacteria (See Fig. 5), the intracellular bacterial growth in the mutant cells was inhibited to similar levels, indicating that the mutant cells impaired some cellular functions commonly associating with chlamydial growth. It has been proposed that pathogenic chlamydiae use different receptors on epithelial mucosa, contributing to the pathogenesis of the LGV and trachoma [36-38]. However, the attachment mechanism of chlamydiae to lymphocytes appears to be very similar, although the variety of strains utilized in our study was limited. Since Jurkat cells did not express heparin at a detectable level [28], this attachment mechanism specific to lymphocytes may be heparin independent.

EMS exposure in mutant cells suggested impairment of cellular function due to a down-regulation of the histone cluster $1 \mathrm{H} 4 \mathrm{c}$, and increased expression of granzyme $\mathrm{K}$ when compared with wild-type cells using DNA microarray analysis with real-time RT-PCR. We also observed irregular over-expression of granzyme K among the mutant cells by immunofluorescence staining, suggesting a possible mechanism refractory of mutant cells to $C$. trachomatis infection. Although further study is necessary to clarify how such molecular alteration was directly involved in a failure of $C$. trachomatis replication, such mutant cells are potentially useful for elucidating the interaction of pathogenic chlamydiae with lymphocytes. In addition, recent works using animals revealed that granzyme was expressed and produced not only in T cells or NK cells, but also in macrophages, although a counterpart in humans is yet to be discovered [41, 42]. These findings may provide a possible explanation as to why pathogenic chlamydiae could not survive effectively in macrophages [43], implying that some macrophages producing granzymes have a critical role for eliminating chlamydiae. 
In conclusion, we have demonstrated that $C$. trachomatis L2 can infect Jurkat cells with lymphoid properties. Although further study is required to clarify the mechanisms involved in C. trachomatis L2 interactions with lymphocytes, this model is potentially valuable for not only offering new insights into host-parasite relationships, but also to study C. trachomatis dissemination into tissues via the movement of lymphocytes.

\section{Materials and methods}

\section{1. Bacteria}

C. trachomatis 434/Bu (LGV: serovar L2) and D/UW3Cx (serovar D) strains was purchased from ATCC (Manassas, VA). Bacteria were propagated in a HEp-2 cell culture system as described previously [34]. In brief, the infected cells were harvested on day 2 and disrupted by freeze-thawing and ultrasonication. After centrifugation to remove cell debris, bacteria were concentrated by high-speed centrifugation. Bacterial pellets were resuspended in sucrose-phosphate-glutamic acid buffer ( $0.2 \mathrm{M}$ sucrose, 3.8 $\mathrm{mM} \mathrm{KH} \mathrm{PO}_{4}, 6.7 \mathrm{mM} \mathrm{Na}_{2} \mathrm{HPO}_{4}, 5 \mathrm{mM}$ L-glutamic acid, $\mathrm{pH}$ 7.4) and then stored at -80 ${ }^{\circ} \mathrm{C}$ for later use. Numbers of infectious progenies were determined as inclusion forming units (IFUs) by counting chlamydial inclusions formed in HEp-2 cells using fluorescein isothiocyanate (FITC)-conjugated monoclonal anti-Chlamydia antibody specific to Chlamydia lipopolysaccharide (LPS) (with Evans Blue) (Denka Seiken Co. Ltd., Tokyo, Japan) [34]. 


\section{2. Cells}

The epithelial cell line, HeLa cells (also HEp-2 cells), and lymphocyte cell line, Jurkat cells, were cultured at $37{ }^{\circ} \mathrm{C}$ in $5 \% \mathrm{CO}_{2}$ in DMEM medium and RPMI1640 medium respectively, each containing $10 \%$ heat-inactivated fetal calf serum (FCS) and antibiotics $(10 \mu \mathrm{g} / \mathrm{ml}$ gentamicin; $10 \mu \mathrm{g} / \mathrm{ml}$ vancomycin; $1 \mu \mathrm{g} / \mathrm{ml}$ amphotericin $\mathrm{B})$ (Sigma, St. Louis, MO). HEp-2 cells were also used to propagate bacteria as described above. We also established random chemical mutagenesis of Jurkat cells using ethymethanesulfonate (EMS) and isolated clones that were resistant to C. trachomatis L2 infection. In brief, Jurkat cells (wild cells) $\left(5 \times 10^{6}\right.$ cells) grown in T-250 flasks were mutagenized for $24 \mathrm{~h}$ with $100 \mu \mathrm{g} / \mathrm{ml}$ of EMS in RPMI1640 medium with FCS. After removal of the EMS solution, surviving Jurkat cells were grown until they reached a concentration of approximately $10^{7}$ cells. C. trachomatis L2 were then used to infect mutated cells for $1 \mathrm{~h}$ at a multiplicity of infection (MOI) of 100. After washing, the culture was maintained by replacing half of the medium at three-day intervals for up to a month. The remaining cells were cloned by limiting dilution, and cells without inclusion formation after $C$. trachomatis $\mathrm{L} 2$ infection were used as mutant cells resistant to the infection, as described in the experiments below. Human peripheral blood monocytes were isolated from whole blood provided by healthy volunteers, by density gradient centrifugation with Histopaque (Sigma). The resulting peripheral blood mononuclear cells were washed three times with Hanks' balanced salt solution (HBSS) and suspended in RPMI 1640 medium containing 10\% FCS. The peripheral blood mononuclear cell suspensions were then dispensed in tissue culture flasks and incubated for $2 \mathrm{~h}$ at $37^{\circ} \mathrm{C}$ in $5 \% \mathrm{CO}_{2}$ to adhere out the monocytes. After incubation, nonadherent cells were collected, washed with HBSS, and resuspended in RPMI 1640 medium 
containing $10 \%$ FCS. Cytocentrifuged preparations of the resulting lymphocyte fractions stained with Giemsa showed greater than $95 \%$ lymphocytes by morphology. Informed consent with written was obtained from all volunteers in this study, and the study was approved by the ethics committee of Faculty of Health Sciences, Hokkaido University.

\section{3. Infection}

HeLa, Jurkat (wild and mutant), enriched human-blood lymphocytes and mutant cells were adjusted to a concentration of $2 \times 10^{5}$ cells/well and infected with bacteria at a MOI of either 1 or 10 by static culture (without centrifugation) for $1 \mathrm{~h}$ at room temperature. After washing to remove non-infected bacteria with Hank's balanced solution (HBSS) (Sigma), infected and uninfected cells at a concentration of $2 \times 10^{5}$ cells/well were incubated for up to seven days in the presence or absence of various drugs (Sigma) [antibiotics: DOX $(0.064 \mu \mathrm{g} / \mathrm{ml})$, AZM $(0.125 \mu \mathrm{g} / \mathrm{ml})$, OFLX $(0.5$ $\mu \mathrm{g} / \mathrm{ml})$; sphingomyelin biosynthesis inhibitors: myriocin $(25 \mu \mathrm{M})$, fumonisin B1 (5 $\mu \mathrm{g} / \mathrm{ml})]$. While the cells were normally cultured for antibiotic experiment in either DMEM or RPMI medium containing $10 \%$ FCS, the cells were incubated for sphingomyelin experiment in the medium containing $0.1 \%$ FCS (to ensure that the medium contains less than sphingomyelin) according to the method described previously [44]. The antibiotics were used at concentrations that inhibited bacterial growth in HeLa cells, as reported previously [45]. The sphingomyelin biosynthesis inhibitors were also used at concentrations previously described [39]. We confirmed that neither these drugs used at each of the working concentrations nor low concentration of 
FCS had any cytotoxic effect on the cells used for up to culture period.

\section{4. Assessment of inclusion formations and bacterial attachment to cells}

For up to two days after infection, cells were collected to determine the morphology of chlamydial inclusions, as described previously [34]. In brief, after the cells were fixed onto a slide with ethanol, they were stained with FITC-conjugated monoclonal anti-Chlamydia antibody specific to Chlamydia lipopolysaccharide (LPS) (with Evans Blue) (Denka Seiken Co. Ltd., Tokyo, Japan). Cells stained with specific antibodies were observed using a conventional [IX71 (Olympus, Tokyo, Japan)] or a confocal laser microscope [LSM510 (Carl Zeiss Japan Group, Tokyo, Japan)]. Bacterial attachment to cells was also estimated immediately after infection by immunofluorescence microscopy using FITC-conjugated monoclonal anti-Chlamydia antibody specific to Chlamydia LPS (without Evans Blue) (Progen Biotechniik, Deutschland, Germany). For bacterial attachment analysis, the cells were washed in cold phosphate-buffered saline (PBS) containing 1\% (w/v) bovine serum albumin (BSA) and then fixed in PBS containing 70\% ethanol. The fixed cells were incubated with FITC-labeled Chlamydia LPS antibody. The percentage of the cells with attached bacteria was also estimated under a fluorescence microscope [IX71 (Olympus)] by counting at least 200 cells in three random fields for each sample.

\section{5. TEM}

TEM was performed in accordance with a method described previously [17]. In brief, bacterial cultures were immersed in a fixative containing $3 \%$ glutaraldehyde in $0.1 \mathrm{M}$ PBS pH 7.4, for $24 \mathrm{~h}$ at $4{ }^{\circ} \mathrm{C}$. After briefly washing with PBS, cells were processed for 
alcohol dehydration and embedded in Epon 812. Ultra-thin sections of cells were stained with lead citrate and uranium acetate before viewing by TEM (Hitachi H7100; Hitachi, Tokyo, Japan).

\section{6. DNA microarray}

Wild and mutant cells were separately incubated with bacteria, before total ribonucleic acid (RNA) was extracted from each culture $10 \mathrm{~h}$ after incubation using an RNeasy Mini Kit (Qiagen, Valencia, CA) according to manufacturer's instructions. Extracted RNA was treated with DNase (DNA-free; Ambion, Austin, TX) to eliminate any contaminating DNA. Polymerase chain reaction, without reverse transcription, was used to confirm the absence of DNA. RNA (50 ng) was amplified by the method with T7 promoter mediated cRNA amplification [46], and the amplified cRNA was also labeled with Cy3 using a Quick Amp Labeling Kit (Agilent Technologies, Santa Clara, CA). The amplified-labeled cRNA was hybridized to a $4 \times 44 \mathrm{~K}$ Whole Human Genome SurePrint G3 gene expression DNA array (Agilent Technologies), and analyzed using a microarray scanner (Agilent Technologies). Hokkaido System Science (Sapporo, Japan) carried out the DNA microarray analysis (also including normalization of gene expressions), except for the RNA preparation and DNase treatment.

\section{7. Real-time RT-PCR}

As mentioned above, total RNA was also extracted from C. trachomatis infected HeLa cells with or without antibiotics, using a RNeasy Mini Kit (Qiagen, Valencia, CA) according to the manufacturer's protocol. Extracted RNA was treated with DNase I (DNA-free; Ambion, Austin, TX) to eliminate contaminating DNA. The resulting RNA 
preparations were confirmed to be DNA-free when a negative result was produced using PCR without the reverse transcription step. RT of $2 \mu \mathrm{g}$ of total RNA by avian myeloblastosis virus reverse transcriptase was performed with random primers in a commercial reaction mixture (Reverse Transcription System; Promega, Madison, WI). Resulting cDNAs were then subjected to PCR with pairs of primers specific for $C$. pneumoniae $16 \mathrm{~S}$ rRNA (sense primer, 5'-GGA CCT TAG CTG GAC TTG ACA TGT-3'; antisense primer, 5'-CCA TGC AGC ACC TGT GTA TCT G-3') [47]. The thermal cycling conditions were $95^{\circ} \mathrm{C}$ for $10 \mathrm{~min}$ and 50 cycles of $95^{\circ} \mathrm{C}$ for $15 \mathrm{~s}, 60^{\circ} \mathrm{C}$ for $1 \mathrm{~min}$, and $72^{\circ} \mathrm{C}$ for $20 \mathrm{~s}$. The profiles of the melting temperatures were assessed for each PCR run for confirmation of the specificities of the PCR products. As a standard for $C$. trachomatis $16 S$ rRNA, a series of diluted $C$. trachomatis DNA extracted from $C$. trachomatis L2 infected HeLa cells (See "4 .3. Infection") was used. The relative concentrations of $C$. trachomatis16S rRNA (number of copies per PCR mixture) were calculated from the standard curve. According to the same procedure, the cDNA prepared from either the wild or the mutant cells with or without C. trachomatis L2 (MOI 10, at $10 \mathrm{~h}$ after infection) were also used for real-time PCR, to confirm gene expression changes observed in the DNA microarray analysis. The specific primers targeting to GZMK (sense primer, 5'-AAA CTT CCA GTC TCC CAC TGT GGT TT-3'; antisense primer, 5'-AAC CTT GCA TTT GGT TCC AG-3'), NT5E (sense primer, 5'-CGC AAC AAT GGC ACA ATT AC-5'; antisense primer, 5'-CTC GAC ACT TGG TGC AAA GA-3'), EOMES (sense primer, 5'-CCA CTG CCC ACT ACA ATG TG-3'; antisense primer, 5'-TTC CCG AAT GAA ATC TCC TG-3'), HIST1H4C (sense primer, 5'-TAA GGT GCT CCG GGA TAA CA-5'; antisense primer, 5'-CCC TGA CGT TTT AGG GCA TA-3'), GAGE7 (sense primer, 5'-GAA CCA GCA ACT CAA 
CGT CA-3'; antisense primer, 5'-TTC ACC TCC TCT GGA TTT GG-3') and MAGEA2B (sense primer, 5'-CTC CAG CAA CCA AGA AGA GG-3'; antisense primer, 5'-CAG CAT TTC TGC CTT TGT GA-3') were picked up from GeneBank cDNA sequences (accession numbers: GZMK, NM002104; NT5E, BC065937; EOMES, NM005442; HIST1H4C, NM003542; GAGE7, NM021123; MAGEA2B, NM153488, respectively) by using the program Primer 3 (available at the website http://www-genome.wi.mit.edu/cgi-bin/primer/primer3.cgi). The primer set targeting to house keeping, and glyceraldehyde 3-phosphate dehydrogenase (GAPDH) (sense primer, 5'-AAC GGG AAG CTC ACT GGC ATG-3'; antisense primer, 5'-TCC ACC AAC CTG TTG CTG TAG-3') [28] was also used. These genes of fold changes were estimated by using $\triangle \Delta \mathrm{CT}$ method based on GAPDH expression, according to the method reported previously [48].

\section{8. Immunofluorescence staining of granzyme $K$ in either the mutant or the wild cells}

The cells were washed in cold PBS containing 5\% (w/v) BSA and then incubated with human CD3 monoclonal antibody (BioLegend, San Diego, CA) for 20 min on ice followed by incubation with FITC-labeled secondary antibody (Santa Cruz Biotechnology Inc., Santa Cruz, CA). After washing with 1\%BSA-PAS, the cells were fixed and increased permeability with $1 \%$ paraformaldehyde containing $0.1 \%(\mathrm{w} / \mathrm{v})$ saponin (Sigma) for $30 \mathrm{~min}$ on ice. After washing with $1 \%$ BSA-PBS, the fixed cells were incubated with anti-human granzyme K antibody (Abcam Inc., Cambrige, UK) for 30 min on ice, and then incubated with Dylight ${ }^{\mathrm{TM}} 549$ - labeled secondary antibody (KPL Inc., Gaithersburg, MA). After staining, granzyme $\mathrm{K}$ in the cells was detected with a fluorescence microscope, Biozero (Keyence, Tokyo, Japan); the percentage of the 
Kubo Tet al

cells with irregular over-expressing granzyme $\mathrm{K}$ was also estimated under the fluorescence microscope by counting at least 200 cells in three random fields for each sample.

\section{9. Statistical analysis}

Statistical analysis was performed using an unpaired Student $t$ test.

\section{Acknowledgment}

This study was supported in part by grants-in-aid for scientific research from KAKENHI. 


\section{References}

[1] Mabey DC, Fraser-Hurt N. Trachoma. BMJ 2001;323: 218-221.

[2] Mabey DC, Solomon AW, Foster A. Trachoma. Lancet 2003;362: 223-229.

[3] Bjartling C, Osser S, Persson K. The frequency of salpingitis and ectopic pregnancy as epidemiologic markers of Chlamydia trachomatis. Acta Obstet Gynecol Scand 2000;79: 123-128.

[4] Toth M, Patton DL, Campbell LA, Carretta EI, Mouradian J, Toth A, Shevchuk M, Baergen $\mathrm{R}$, Ledger W. Detection of chlamydial antigenic material in ovarian, prostatic, ectopic pregnancy and semen samples of culture-negative subjects. Am J Reprod Immunol 2000;43: 218-222.

[5] Mabey D, Peeling RW. Lymphogranuloma venereum. Sex Transm Infect 2002;78: $90-92$.

[6] Nieuwenhuis RF, Ossewaarde JM, Hannelore M, Götz HM, Jan Dees J, Thio HB, Thomeer MG, den Hollander JC, Neumann MH, van der Meijden WI. Resurgence of lymphogranuloma venereum in Western Europe: an outbreak of Chlamydia trachomatis serovar L2 proctitis in The Netherlands among men who have sex with men. Clin Infect Dis 2004;39: 996-1003.

[7] Meyer T, Arndt R, von Krosigk A, Plettenberg A. Repeated detection of lymphogranuloma venereum caused by Chlamydia trachomatis L2 in homosexual men in Hamburg. Sex Transm Infect 2005;81: 91-92.

[8] Herida M, Sednaoui P, Couturier E, Scieux C, Lemarchand N, Kreplak G, Clerc M, Timsit J, Goulet V, Desenclos JC. Semaille, Rectal lymphogranuloma venereum, France. Emerg Infect Dis 2005;11: 505-506. 
[9] Macdonald N, Ison C, Martin I, Alexander S, Lowndes C, Simms I, Ward H. Initial results of enhanced surveillance for lymphogranuloma venereum (LGV) in England. Euro Surveill 2005;10: E0501275.

[10] Kropp RY, Wong T. Emergence of lymphogranuloma venereum in Canada. CMAJ 2005;172: 1674-1676.

[11] Stark D, van Hal S, Hillman R, Harkness I, Marriott D, Lymphogranuloma venereum in Australia: anorectal Chlamydia trachomatis serovar L2b in men who have sex with men. J Clin Microbiol 2007;45:1029-1031.

[12] Perine PL, Stamm WE. Lymphogranuloma venereum, in Holmes KK (Ed.), Sexually Transmitted Diseases: MCG (Manual), New York, McGraw-Hill, 1998, pp. 423-432.

[13] Matsumoto A, Higashi N, Tamura A. Electron microscope observation on the effects of polymixin B sulfate on cell walls of Chlamydia psittaci. J Bacteriol $1973 ; 113: 357-364$.

[14] Matsumoto A, Manire GP, Electron microscopic observations on the fine structure of cell walls of Chlamydia psittaci. J Bacteriol 1970;104: 1332-1337.

[15] Rockey DD, Matsumoto A. The chlamydial developmental cycle, in Brun YV, Shimkets LL (eds), Prokaryotic Development, ASM Press, Washington D.C, 2000, pp.403-425.

[16] Haranaga S, Yamaguchi H, Friedman H, Izumi S, Yamamoto Y. Chlamydia pneumoniae infects and multiplies in lymphocytes in vitro. Infect Immun 2001;69: $7753-7759$.

[17] Yamaguchi H, Haranaga S, Friedman H, Moor JA, Muffly KE, Yamamoto Y. A Chlamydia pneumoniae infection model using established human lymphocyte cell 
lines. FEMS Microbiol Lett 2002;216: 229-234.

[18] Kaul R, Uphoff J, Wiedeman J, Yadlapalli S, Wenman WM. Detection of Chlamydia pneumoniae DNA in $\mathrm{CD}^{+}$lymphocytes from healthy blood donors and patients with coronary artery disease. Circulation 2000;102: 2341-2346.

[19] Dessus-Babus S, Moore CG, Whittimore JD, Wyrick PB, Comparison of Chlamydia trachomatis serovar L2 growth in polarized genital epithelial cells grown in three-dimensional culture with non-polarized cells. Microbes Infect 2008;10: 563-570.

[20] Lambden PR, Pickett MA, Clarke IN. The effect of penicillin on Chlamydia trachomatis DNA replication. Microbiology 2006;152: 2573-2578.

[21] Al-Younes HM, Brinkmann V, Meyer TF. Interaction of Chlamydia trachomatis serovar L2 with the host autophagic pathway. Infect Immun 2004;72: 4751-62.

[22] Yamaguchi H, Friedman H, Yamamoto M, Yasuda K, Yamamoto Y. Chlamydia pneumoniae resists antibiotics in lymphocytes. Antimicrob Agents Chemother 2003;47: 1972-1975.

[23] Carabeo RA, Mead DJ, Hackstadt T. Golgi-dependent transport of cholesterol to the Chlamydia trachomatis inclusion. Proc Natl Acad Sci USA 2003;100: 6771-6776.

[24] van Ooij C, Kalman L, van Ijzendoorn, Nishijima M, Hanada K, Mostov K, Engel JN. Host cell-derived sphingolipids are required for the intracellular growth of Chlamydia trachomatis. Cell Microbiol 2000;2: 627-637.

[25] Robertson DK, Gu L, Rowe RK, Beatty WL. Inclusion biogenesis and reactivation of persistent Chlamydia trachomatis requires host cell sphingolipid biosynthesis. PLoS Pathog 2009;5: e1000664. 
[26] Yamaguchi H, Matsuo J, Sugimoto S, Utsumi M, Yamamoto Y. Inhibition of lymphocyte CD3 expression by Chlamydophila pneumoniae infection. Microb Pathog 2008;45: 290-296.

[27] Hirai I, Utsumi M, Yamaguchi H, Yamamoto Y. Chlamydia pneumoniae infection suppresses Staphylococcus enterotoxin B-induced proliferation associated with down-expression of CD25 in lymphocytes. Can J Microbiol 2010;56: 289-294.

[28] Kobayashi M, Ishida K, Matsuo J, Nakamura S, Nagasawa A, Motohashi K, Yao T, Hirai I, Yamamoto Y, Suzuki H, Shimizu C, Matsuno K, Yamaguchi H. Chlamydophila pneumoniae attachment and infection in low proteoglycan expressing human lymphoid Jurkat cells. Microb Pathog 2011;51: 209-216.

[29] Dosch HM, White D, Grant C. Reconstitution of nude mouse T cell function in vivo: IL 2-independent effect of human T cells. J Immunol 1985;134: 336-342.

[30] Azenabor AA, Cintrón-Cuevas J, Schmitt H, Bumah V. Chlamydia trachomatis induces anti-inflammatory effect in human macrophages by attenuation of immune mediators in Jurkat T-cells. Immunobiology 2011;216:1248-1255.

[31] Hackstadt T, Rockey DD, Heinzen RA, Scidmore MA. Chlamydia trachomatis interrupts an exocytic pathway to acquire endogenously synthesized sphingomyelin in transit from the Golgi apparatus to the plasma membrane. EMBO J 1996;15: 964-977.

[32] Hackstadt T, Scidmore MA, Rockey DD. Lipid metabolism in Chlamydia trachomatis-infected cells: directed trafficking of Golgi-derived sphingolipids to the chlamydial inclusion. Proc Natl Acad Sci USA 1995;92: 4877-4881.

[33] Matsuo J, Kobayashi M, Nakamura S, Mizutani Y, Yao T, Hirai I, Yamamoto Y, Yamaguchi H. Stability of Chlamydophila pneumoniae in a harsh environment 
without a requirement for acanthamoebae. Microbiol Immunol 2010;54: 63-73.

[34] Roblin PM, Dumornay W, Hammerschlag MR. Use of HEp-2 cells for improved isolation and passage of Chlamydia pneumoniae. J Clin Microbiol 1992;30: 1968-1971.

[35] Kutlin A, Flegg C, Stenzel D, Reznik T, Roblin PM, Mathews S, Timms P, Hammerschlag MR. Ultrastructural study of Chlamydia pneumoniae in a continuous-infection model. J Clin Microbiol 2001;39: 3721-3723.

[36] Beatty WL, Byrne GI, Morrison RP. Morphologic and antigenic characterization of interferon gamma-mediated persistent Chlamydia trachomatis infection in vitro. Proc Natl Acad Sci USA 1993;90: 3998-4002.

[37] Kutlin A, Roblin PM, Hammerschlag MR. In vitro activities of azithromycin and ofloxacin against Chlamydia pneumoniae in a continuous-infection model. Antimicrob Agents Chemother 1999;43: 2268-2272.

[38] Carabeo RA, Hackstadt T. Isolation and characterization of a mutant Chinese hamster ovary cell line that is resistant to Chlamydia trachomatis infection at a novel step in the attachment process. Infect Immun 2001;69: 5899-5904.

[39] Robertson DK, Gu L, Rowe RK, Beatty WL. Inclusion biogenesis and reactivation of persistent Chlamydia trachomatis requires host cell sphingolipid biosynthesis. PLoS Pathog 2009;5: e1000664.

[40] Derré I, Swiss R, Agaisse H. The Lipid Transfer Protein CERT Interacts with the Chlamydia Inclusion Protein IncD and Participates to ER-Chlamydia Inclusion Membrane Contact Sites. PLoS Pathog 2011;7: e1002092.

[41] Joeckel LT, Wallich R, Martin P, Sanchez-Martinez D, Weber FC, Martin SF, Borner C, Pardo J, Froelich C, Simon MM. Mouse granzyme K has 
pro-inflammatory potential. Cell Death Differ 2011;18: 1112-1119.

[42] Baba T, Ishizu A, Iwasaki S, Suzuki A, Tomaru U, Ikeda H, Yoshiki T, Kasahara M. $\mathrm{CD}^{+} / \mathrm{CD}^{+}$macrophages infiltrating at inflammatory sites: a population of monocytes/macrophages with a cytotoxic phenotype. Blood 2006;107: 2004-2012.

[43] Wolf K, Fischer E, Hackstadt T. Degradation of Chlamydia pneumoniae by peripheral blood monocytic cells. Infect Immun 2005;73: 4560-4570.

[44] van Ooij C, Kalman L, van Ijzendoorn, Nishijima M, Hanada K, Mostov K, Engel JN. Host cell-derived sphingolipids are required for the intracellular growth of Chlamydia trachomatis. Cell Microbiol 2000;2:627-637.

[45] Suchland RJ, Geisler WM, Stamm WE. Methodologies and cell lines used for antimicrobial susceptibility testing of Chlamydia spp. Antimicrob Agents Chemother 2003;47: 636-642.

[46] van Bakel H, van Werven FJ, Radonjic M, Brok MO, van Leenen D, Holstege FC, Timmers HT. Improved genome-wide localization by ChIP-chip using double-round T7 RNA polymerase-based amplification. Nucleic Acids Res 2008;36: e21.

[47] Berger M, Schroder B, Daeschlein G, Schneider W, Busjahn A, Buchwalow I, Luft FC, Haller H. Chlamydia pneumoniae DNA in non-coronary atherosclerotic plaques and circulating leukocytes. J Lab Clin Med 2000;136:194-200.

[48] Livak KJ, Schmittgen TD. Analysis of relative gene expression data using real-time quantitative PCR and the 2(-Delta Delta C(T)) Method. Methods 2001;25: 402-408. 
Kubo Tet al

\section{Legends to figures}

Fig. 1. One-step growth curves for C. trachomatis in Jurkat cells (A) and HeLa cells (B) and fluorescent microscope images of $C$. trachomatis L2-infected Jurkat cells (C). Both cell types were infected with bacteria at a MOI of either 1 or 10 by static culture, and incubated for up to eight days. (A, B) Number of infectious progenies in cultures post-infection. Cell lysates prepared at each time point were inoculated onto HEp-2 cell monolayers to assess the number of infective progeny. Number of inclusions was determined by staining with FITC-conjugated anti-Chlamydia antibody. Data shown represent the mean + standard deviation (SD) (error bars), obtained from at least three independent experiments performed in triplicate. ${ }^{*} p<0.05$ versus the value immediately after infection (0 days). (C) Representative fluorescence microscope images showing inclusion formation. Infected cells were stained with FITC-conjugated anti-Chlamydia antibody. Magnification, $\times 400$ [0-48 h: with a conventional microscope (Olympus IX71)] or $\times 1,000$ [right image, 48 h: with a confocal microscope (Carl Zeiss LSM510)].

Fig. 2. Representative TEM images showing C. trachomatis L2 inclusion formation and bacterial morphological traits in Jurkat cells at $48 \mathrm{~h}$ post-infection (MOI 10, static infection). Inclusion formation of $C$. trachomatis L2 in Jurkat cells, showing the bacteria packed within an inclusion. EB, representative elementary bodies. RB, representative reticular bodies. AB, representative aberrant bodies. Scale, $2 \mu \mathrm{m}$. 
Fig. 3. Increases of infectious progenies for C. trachomatis in enriched human-blood lymphocytes obtained from three healthy donors. The cells were infected with bacteria at a MOI 10 by static culture, and incubated for up to three days. Number of infectious progenies in cultures post-infection was estimated by the IFU assay (See Fig. 1 legend). Plots show each of average values with SD (bar). ${ }^{*} p<0.05$ versus the value immediately after infection (0 days).

Fig. 4. Effect of antibiotics on the growth of C. trachomatis L2 in HeLa cells and Jurkat cells. Both cell types were infected with bacteria at a MOI of 10 by static culture, and incubated for up to three days in the presence or absence of antibiotics (DOX, AZM, OFLX). (A) Number of infectious progenies in cultures post-infection in the presence or absence of antibiotics. Cell lysates prepared at each time point were inoculated onto HEp-2 cell monolayers to assess infective progeny. Number of inclusions was determined by staining with FITC-conjugated anti-Chlamydia antibody. The data shown represent the mean $+\mathrm{SD}$ (error bars), obtained from at least three independent experiments performed in triplicate. $* p<0.05$ versus the control value without antibiotics at the same time point. (B) Representative fluorescence microscope images showing inclusion formation. Infected cells were stained with FITC-conjugated anti-Chlamydia antibody and observed under a fluorescent microscopy [IX71 (Olympus)]. Green, chlamydial inclusion formation. Red, cells (Evans blue). Magnification, $\times 200($ Scale, $100 \mu \mathrm{m})$. (C) C. trachomatis 16S rRNA transcription levels in either HeLa or Jurkat cells treated with or without antibiotics at 3 days after the infection estimated by real-time RT-PCR. The relative concentrations of $C$. trachomatis 
$16 S$ rRNA (number of copies per PCR mixture) were calculated from the standard curve, as described in the Materials and Methods. The data shown represent the means + SD, obtained from at least three independent experiments performed in triplicate. ${ }^{*} p<0.05$, significantly different from each data without the treatment [left side, (-)].

Fig.5. Effect of host cell sphingomyelin biosynthesis inhibition on C. trachomatis growth in HeLa cells and Jurkat cells. Both cell types were infected with bacteria at a MOI of 10 by static culture, and incubated for up to three days in the presence or absence of sphingomyelin biosynthesis inhibitors (myriocin, fumonisin B1). (A) Representative fluorescent microscope images showing inclusion formation. Infected cells were stained with FITC-conjugated anti-Chlamydia antibody and observed under a fluorescence microscope [IX71 (Olympus)]. Magnification, $\times 200$ (Scale, 100 $\mu \mathrm{m}) .(\mathrm{B}$, C) Number of infectious progenies in the cultures in the presence or absence of the inhibitors after infection. Cell lysates prepared at each time point were inoculated onto HEp-2 cell monolayers to assess infective progeny. B, HeLa. C, Jurkat. Number of inclusions was determined by staining with FITC-conjugated anti-Chlamydia antibody. Data shown represent the mean + SD (error bars), obtained from at least three independent experiments performed in triplicate. ${ }^{*} p<0.05$ versus the control value without the inhibitors at the same time point.

Fig. 6. Attachment and replication of C. trachomatis in mutant cells derived from Jurkat cells. Both mutant and wild cells were infected with bacteria at a MOI of 10 by static 
culture, and incubated for up to three days. (A) Representative fluorescent microscope images showing C. trachomatis L2 inclusion formation with a secondary infection in the mutant and wild cells three days post-infection. Cells were stained with FITC-conjugated anti-Chlamydia antibody and observed under a fluorescent microscopy [IX71 (Olympus)]. Magnification, $\times 200$ (Scale, 100 $\mu$ m). (B) Infectious rate change in mutant and wild cells. The percentage of the cells with attached bacteria was estimated at immediately after infection using a fluorescence microscope [IX71 (Olympus)] (See the Materials and Methods). The data shown represent the mean + SD (error bars), obtained from at least three independent experiments performed in triplicate. (C and D) Number of infectious progenies in wild and mutant cell cultures (see Fig. 3 legend). C, C. trachomatis L2. D, C. trachomatis D. The data shown represent the mean $+\mathrm{SD}$ (error bars), obtained from at least three independent experiments performed in triplicate. ${ }^{*} p<0.05$ versus the value of the wild cells at the same time point.

Fig. 7. Relative gene expression changes of the C. trachomatis-infected mutant cells with over-expression [ $>2.0$ fold (A)] and down-expression $[<1.0$ fold (B)], as compared with those of wild type cells infected with the bacteria (at $10 \mathrm{~h}$ after infection). See the Materials and Methods. GZMK, granzyme K. NT5E, 5'-nucleotidase, ecto (CD73). EOMES, eomesodermin. MLC1, megalencephalic leukoencephalopathy with subcortical cysts. GAGE7, Cancer/Testis Antigens, HIST1H4C, histone cluster 1, H4c. 
Kubo T et al

Fig. 8. Quantitative real-time RT-PCR analysis of transcripts with specific change in either mutant or wild cells with or without infection (at 10h after infection). Gene abbreviation, see the legend of Fig. 6. The relative gene expressions are indicated as fold changes of each of the samples as compared with wild cells without infection as estimated in the Materials and Methods. The data shown represent the means $+\mathrm{SD}$, obtained from at least three independent experiments performed in triplicate. ${ }^{*} p<0.05$, significantly different from each data of wild cells without infection.

Fig. 9. Irregular over-expression of granzyme K among mutant cells as compared with wild cells. Asterisks show granzyme K irregular over-expression among mutant cells. Green, CD3 expression. Orange, granzyme K expression. White dished square are also enlarged below (Enlarged). Magnification, $\times 400$. 
Fig. 1.

A

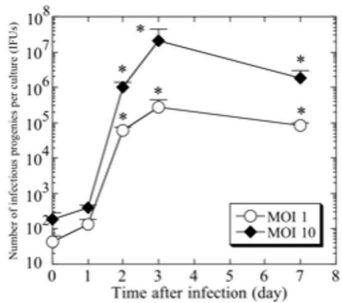

B

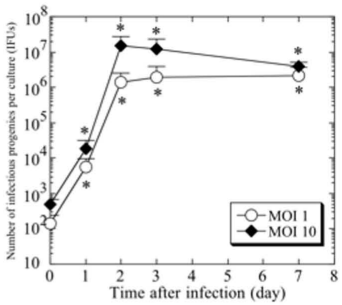

C
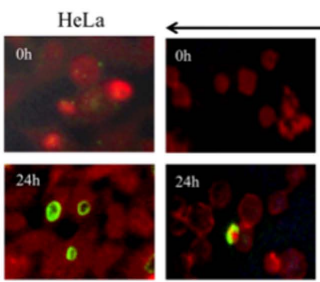

24h

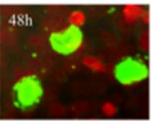

$48 \mathrm{~h}$

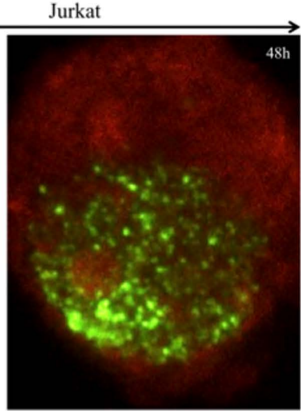


Fig. 2.

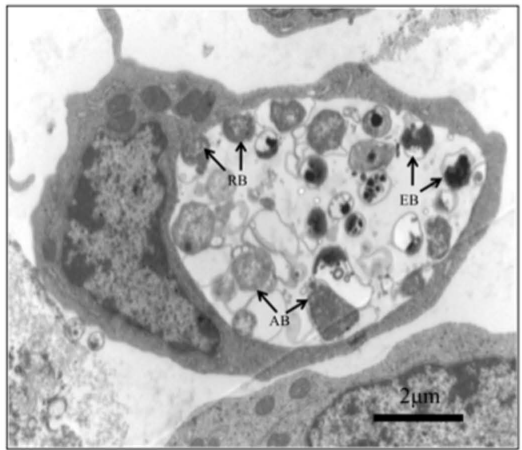


Fig. 3.

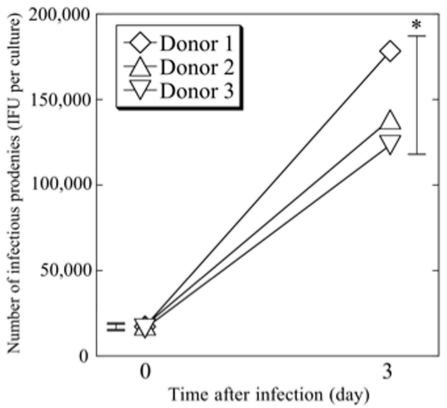


Fig. 4.

A

HeLa

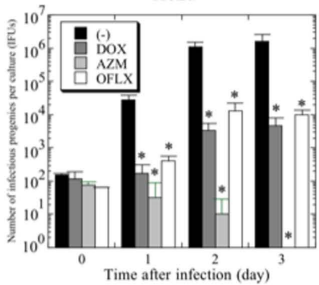

B
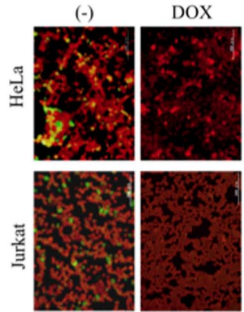

C

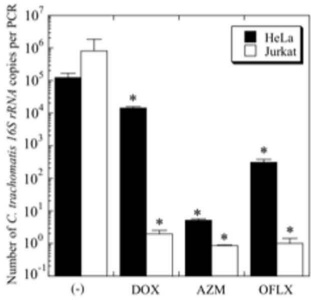

Jurkat

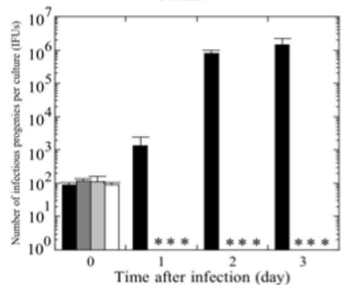

OFLX

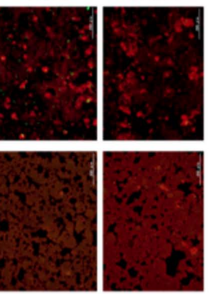


Fig. 5.

A
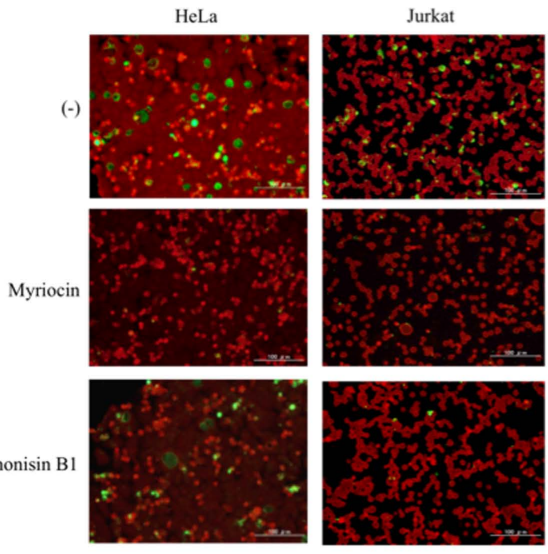

Fumonisin B

B
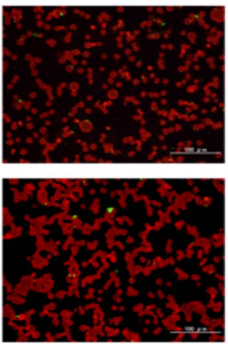

C
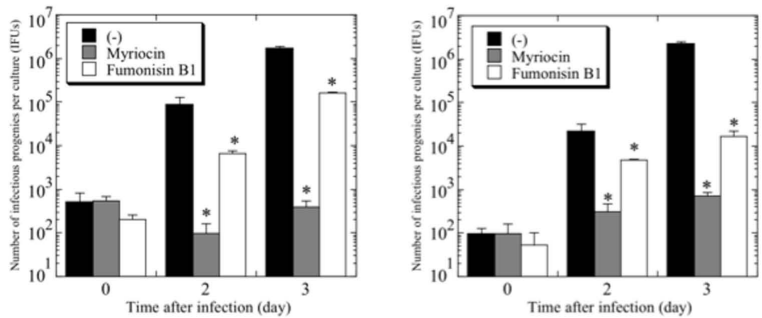
Fig. 6.

A
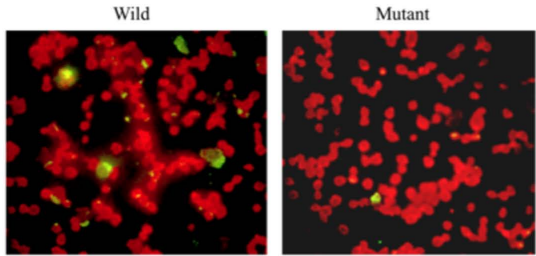

B

C
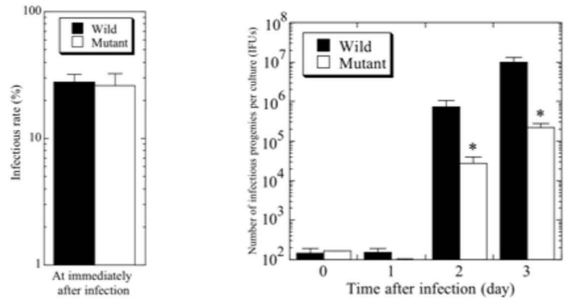

D

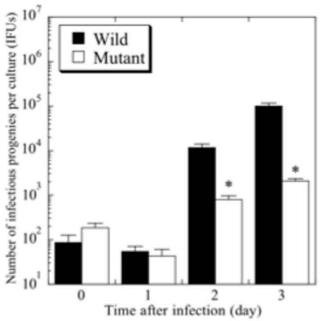


Fig. 7.

A

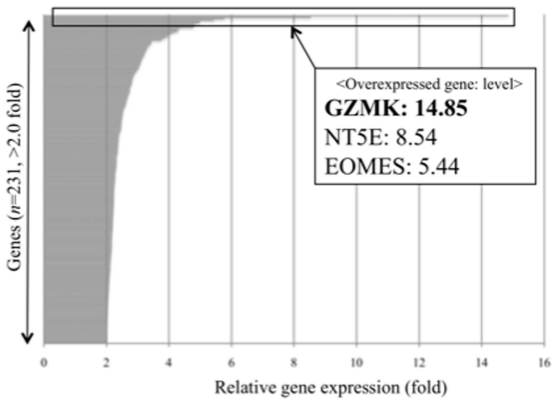

B

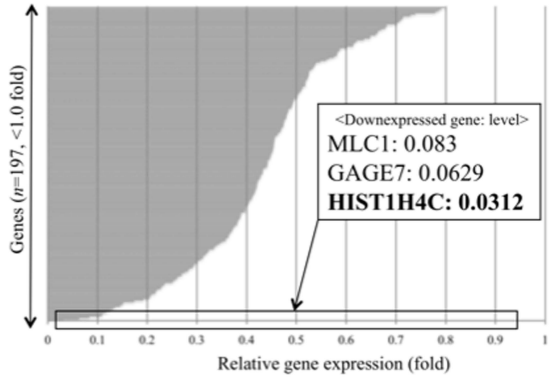


Fig. 8.

A

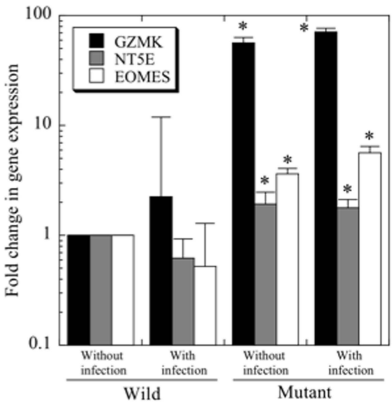

B

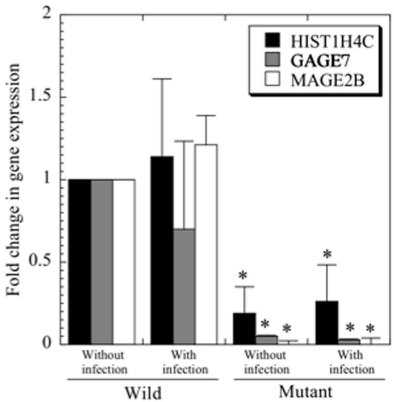


Fig. 9.
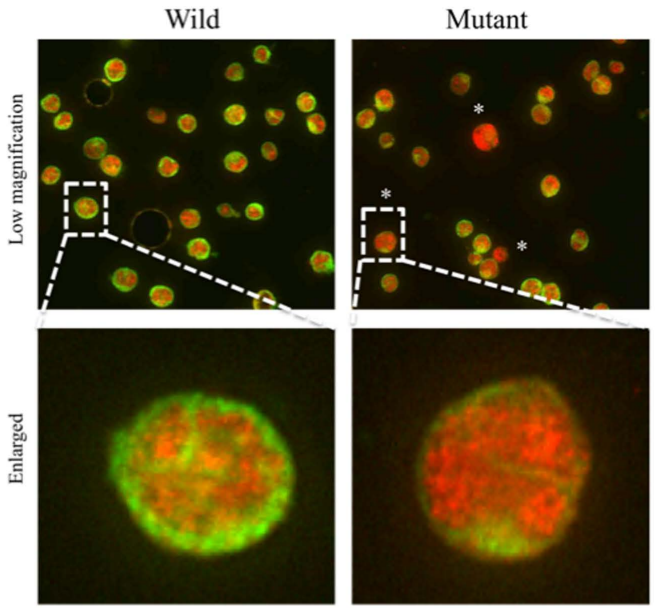